\title{
MINRES Seed Projection Methods for Solving Symmetric Linear Systems with Multiple Right-Hand Sides
}

\author{
Xin Li, ${ }^{1}$ Hao Liu, ${ }^{2}$ and Jingfu $\mathrm{Zhu}^{3}$ \\ ${ }^{1}$ College of Science, Heilongjiang Bayi Agricultural University, Daqing 163319, China \\ ${ }^{2}$ College of Science, Nanjing University of Aeronautics and Astronautics, Nanjing 210016, China \\ ${ }^{3}$ College of Information Technology, Heilongjiang Bayi Agricultural University, Daqing 163319, China
}

Correspondence should be addressed to Hao Liu; hliu@nuaa.edu.cn

Received 14 April 2014; Revised 11 May 2014; Accepted 12 May 2014; Published 12 June 2014

Academic Editor: K. M. Liew

Copyright (C) 2014 Xin Li et al. This is an open access article distributed under the Creative Commons Attribution License, which permits unrestricted use, distribution, and reproduction in any medium, provided the original work is properly cited.

We consider the MINRES seed projection method for solving multiple right-hand side linear systems $A X=B$, where $A \in R^{n \times n}$ is a nonsingular symmetric matrix, $B \in R^{n \times p}$. In general, GMRES seed projection method is one of the effective methods for solving multiple right-hand side linear systems. However, when the coefficient matrix is symmetric, the efficiency of this method would be weak. MINRES seed projection method for solving symmetric systems with multiple right-hand sides is proposed in this paper, and the residual estimation is analyzed. The numerical examples show the efficiency of this method.

\section{Introduction}

Consider the multiple right-hand side linear systems

$$
A X=B,
$$

where $A \in R^{n \times n}$ is a nonsingular symmetric matrix, and $B \epsilon$ $R^{n \times p}$.

Equation (1) plays an important role in chemistry, electronics, structures, control, and other problems; see $[1,2]$ for detail.

In the last few years, the block methods have been developed to solve (1), such as the block conjugate gradient algorithm (BCG) [3], the block generalized minimal residual method [4-6], the block BiCGSTAB method [7], the block QMR method [8], the block least squares method [9], the block Lanczos method [10], and the block IDR $(s)$ method [11] which have been proposed recently. In general, the block methods are faster than solving each one separately.

The meshless methods are extensively used for solving (1); these meshless methods show to be efficient and accurate in terms of their numerical results; see [12-18]. The global methods $[19,20]$ are also a class of important methods. Following the work [20], many other global methods have been developed, including the global BiCG and global BiCGSTAB methods [21], the global Hessenberg and global CMRH methods [22], and the polynomial preconditioned global CMRH method [23, 24]. Generally, the global methods are more appropriate for large and sparse systems.

In many practical applications, the right-hand sides are not arbitrary and are very close; then the seed projection methods are often used to solve (1); see $[1,2,25,26]$. The main idea of this method is selecting one system to be the seed systems firstly, then solving the seed systems by some Krylov subspace method, and creating a Krylov subspace $K$, then projecting the residual of the other systems, called nonseed systems, onto this Krylov subspace $K$ to get the approximate solutions. The process is repeated with other seed systems until all the systems are solved. The seed projection methods were proposed by Smith et al. [1] for the CG method firstly. When $A$ is unsymmetric, Simoncini and Gallopulos [2] proposed the GMRES seed projection method. Later, a seed method which uses Morgan's Krylov subspace augmented with eigenvectors was presented in [25]. Moreover, the seed method can be used to solve unsymmetric shifted systems with multiple right-hand sides [26]. However, if $A$ is a symmetric matrix, the efficiency of these methods would be weak. 
In this paper, we propose the MINRES seed projection method for solving symmetric systems with multiple righthand sides, and the residual estimation is analyzed.

The paper is organized as follows. In Section 2, we give a quick overview of the GMRES seed projection method. In Section 3, we present the MINRES seed projection method and the residual estimation. In the last section, we show the efficiency of our method by numerical experiments.

\section{GMRES Seed Projection Method}

In this section, we recall the GMRES seed projection method for solving (1). Details of the algorithm can be found in [2, 25]. We summarize it in the following algorithm.

Algorithm $1\left(X=\operatorname{Gseed}\left(A, B, X^{(0)}, \mathcal{\varepsilon}, m\right)\right)$. We have the following:

(1) $X=X^{(0)}$;

(2) $R=B-A X$;

(3) for $l=1,2, \ldots, p$ until all the systems are solved

(4) $\left[\sigma, r_{\sigma}\right]=\operatorname{SEED}(R), \beta=\left\|r_{\sigma}\right\|_{2}$;

(5) for $k=1,2, \ldots$, until convergence;

(6) $\left[V_{m+1}, H\right]=\operatorname{Arnoldi}\left(A, r_{\sigma}\right)$;

(7) $\widehat{b_{\sigma}}=\beta e_{1}$, where $e_{1}=(1,0, \ldots, 0)^{T} \in R^{m+1}$;

(8) $\widehat{b}_{j}=V_{m+1}^{\mathrm{T}}\left(b_{j}-A x_{j}\right), j=1, \ldots, p, j \neq \sigma$;

(9) compute $y_{j}$ by minimizing $\left\|H y-\widehat{b_{j}}\right\|_{2}, y \in R^{m}, j=$ $1, \ldots, p$

(10) $\bar{X}=X+V_{m} Y$, where $Y=\left[y_{1}, \ldots, y_{p}\right]^{\mathrm{T}}$;

(11) $\bar{R}=B-A \bar{X}, \bar{r}(j)=\|\bar{R}(:, j)\|_{2}$;

(12) if $\|\bar{r}(j)\|_{2}<\varepsilon$, then delete the $j$ th systems, let $w$ be the number of the $j$ conforming to this condition, and then $p:=p-w$;

(13) if $\left\|\bar{r}_{\sigma}\right\|_{2}<\varepsilon$, then delete these seed systems, set $p:=$ $p-1$, and go to 3 ;

(14) end(k);

(15) end(l).

We now make a few descriptions of Algorithm 1. Firstly, we give an initial approximation to the solutions $X^{(0)}$, compute the initial residuals, and select seed systems by a function SEED. In this algorithm, SEED applied to the $R$ returns $\sigma$ and $r_{\sigma}$, where $\sigma$ is the index of column of $R$ having the maximum norm. Secondly, we apply restarted GMRES method for solving the seed systems and function Arnoldi applies the Arnoldi procedure to generate an orthogonal basis $V_{m+1}=$ $\left[v_{1}, \ldots, v_{m}\right]$ for the Krylov subspace $K_{m+1}\left(A, r_{\sigma}\right)$. Meanwhile, the nonseed solutions are approximated by projecting the residual $r_{j}=b_{j}-A x_{j}$ on $K_{m+1}\left(A, r_{\sigma}\right)$ and solving the least square problem $\min _{y_{j} \in R^{m}}\left\|H y_{j}-V_{m+1}^{\mathrm{T}} r_{j}\right\|_{2}$. Thirdly, after the seed systems are solved to desired accuracy, new seed systems are selected from the unsolved systems and then the whole procedure is repeated until all the systems are solved. Some theoretical analysis about the above algorithm can be found in $[2,25]$.

\section{MINRES Seed Projection Method}

Based on the MINRES method for solving the symmetric linear systems, in this section, we combine the GMRES seed projection method and the MINRES method and propose the MINRES seed projection method for solving (1). The Arnoldi procedure in GMRES seed projection method is exchanged by Lanczos procedure, and applying the seed projection idea to MINRES method, the MINRES seed projection method is proposed as the following algorithm.

Algorithm $2\left(X=\operatorname{Mseed}\left(A, B, X^{(0)}, \varepsilon, m\right)\right)$. We have the following:

(1) $X=X^{(0)}$;

(2) $R=B-A X$;

(3) for $l=1,2, \ldots, p$ until all the systems are solved;

(4) $\left[\sigma, r_{\sigma}\right]=\operatorname{SEED}(R), \beta=\left\|r_{\sigma}\right\|_{2}$;

(5) for $k=1,2, \ldots$, until convergence;

(6) $\left[Q_{m+1}, \widetilde{T}_{m}\right]=\operatorname{Lanczos}\left(A, r_{\sigma}\right)$;

(7) $\widehat{b_{\sigma}}=\beta e_{1}$, where $e_{1}=(1,0, \ldots, 0)^{T} \in R^{m+1}$;

(8) $\widehat{b}_{j}=V_{m+1}^{\mathrm{T}}\left(b^{(j)}-A x^{(j)}\right), j=1, \ldots, p, j \neq \sigma$;

(9) compute $d^{(j)}$ by minimizing $\left\|\widetilde{T}_{m} d-\widehat{b_{j}}\right\|_{2}, d \in$ $R^{m}, j=1, \ldots, p$;

(10) $\widetilde{X}=X+Q_{m} D$, where $D=\left[d^{(1)}, \ldots, d^{(p)}\right]^{\mathrm{T}}$;

(11) $\widetilde{R}=B-A \widetilde{X}, \widetilde{r}^{(j)}=\|\widetilde{R}(:, j)\|_{2}$;

(12) if $\left\|\widetilde{r}^{(j)}\right\|_{2}<\varepsilon$, then delete $j$ th system, let $w$ be the number of the $j$ conforming to this condition, and then $p:=p-w$;

(13) if $\left\|\widetilde{r}_{\sigma}\right\|_{2}<\varepsilon$, then delete this seed systems, set $p:=$ $p-1$, go to 3 ;

(14) end(k);

(15) end(1).

We now make a few descriptions about Algorithm 2. Firstly, we give an initial approximation to the solutions $X^{(0)}$, compute the initial residuals, and select seed systems by a function SEED. In this algorithm, SEED applied to the $R$ returns $\sigma$ and $r_{\sigma}$, where $\sigma$ is the index of column of $R$ having the maximum norm. Secondly, we apply restarted Lanczos method for solving the seed systems and function Lanczos applies the Lanczos procedure to generate an orthogonal basis $Q_{m+1}=\left[q_{1}, \ldots, q_{m}\right]$ for the Krylov subspace $K_{m+1}\left(A, r_{\sigma}\right)$. Meanwhile, the nonseed solutions are approximated by projecting the residual $r^{(j)}=b^{(j)}-A x^{(j)}$ on $K_{m+1}\left(A, r_{\sigma}\right)$ and solving the least square problem $\min _{d^{(j)} \in R^{m}}\left\|\widetilde{T}_{m} d^{(j)}-Q_{m+1}^{\mathrm{T}} r^{(j)}\right\|_{2}$. Thirdly, after the seed systems are solved to desired accuracy, new seed systems are selected from the unsolved systems and then the whole procedure is repeated until all the systems are solved. 
According to Algorithm 2, we can get

$$
\begin{aligned}
\widetilde{r}^{(j)} & =b^{(j)}-A \tilde{x}^{(j)}=b^{(j)}-A\left(x^{(j)}+Q_{m} d^{(j)}\right) \\
& =r^{(j)}-A Q_{m} d^{(j)}=r^{(j)}-Q_{m+1} \widetilde{T}_{m} d^{(j)}
\end{aligned}
$$

and $Q_{m+1}^{T} \widetilde{r}^{(j)}=Q_{m+1}^{T} r^{(j)}-\widetilde{T}_{m} d^{(j)}$. In addition, let $P=$ $Q_{m+1} Q_{m+1}^{T}$ be the orthogonal operator on $K_{m+1}\left(A, r^{(s)}\right)$. It follows that

$$
\begin{aligned}
\widetilde{r}^{(j)} & =r^{(j)}-A Q_{m} d^{(j)}=(I-P) r^{(j)}+\left(P r^{(j)}-A Q_{m} d^{(j)}\right) \\
& =(I-P) r^{(j)}+Q_{m+1}\left(Q_{m+1}^{T} r^{(j)}-\widetilde{T}_{m} d^{(j)}\right) .
\end{aligned}
$$

The following property can be attained.

Property 1. The residual $\tilde{r}^{(j)}$ in nonseed systems in Algorithm 2 satisfy

$$
\begin{gathered}
\left\|Q_{m+1}^{T} \widetilde{r}^{(j)}\right\|_{2}=\min _{x \in x^{(j)}+K_{m}}\left\|Q_{m+1}^{T}\left(b^{(j)}-A x\right)\right\|_{2}, \\
\left\|\widetilde{r}^{(j)}\right\|_{2}^{2}=\left\|(I-P) r^{(j)}\right\|_{2}^{2}+\min _{d \in R^{m}}\left\|Q_{m+1}^{T} r^{(j)}-\widetilde{T}_{m} d\right\|_{2}^{2} .
\end{gathered}
$$

Theorem 3. Set $K_{m} \equiv K_{m}\left(A, r^{(s)}\right)$ as m-dimension Krylov subspace; one has

(1) for the seed systems: $\tilde{x}^{(s)} \in\left\{x^{(s)}\right\}+K_{m}, \widetilde{r}^{(s)} \in K_{m+1}$, and $\tilde{r}^{(s)} \perp A K_{m} \subseteq K_{m+1} ;$

(2) for the nonseed systems: $\tilde{x}^{(j)} \in\left\{x^{(j)}\right\}+K_{m}, \widetilde{r}^{(j)} \in\left\{r^{(j)}\right\}+$ $K_{m+1}$, and $\widetilde{r}^{(j)} \perp A K_{m}$.

Proof. (1) According to the Lanczos method, the conclusion of (1) is right obviously; that

(2) since $\tilde{x}^{(j)}=\left\{x^{(j)}\right\}+Q_{m} d^{(j)} \in\left\{x^{(j)}\right\}+K_{m}$, it follows

$$
\tilde{r}^{(j)}=b^{(j)}-A \tilde{x}^{(j)}=r^{(j)}-A Q_{m} d^{(j)}=r^{(j)}-Q_{m+1} \widetilde{T}_{m} d^{(j)}
$$

Using $A Q_{m}$ as inner product, we can get

$$
\begin{aligned}
\left(A Q_{m}\right)^{T} \widetilde{r}^{(j)} & =\widetilde{T}_{m}^{T} Q_{m+1}^{T}\left(r^{(j)}-Q_{m+1} \widetilde{T}_{m} d^{(j)}\right) \\
& =\widetilde{T}_{m}^{T}\left(Q_{m+1}^{T} r^{(j)}-\widetilde{T}_{m} d^{(j)}\right) .
\end{aligned}
$$

Since $d^{(j)}=\arg \min _{d \in R^{m}}\left\|Q_{m+1}^{T} r^{(j)}-\widetilde{T}_{m} d\right\|_{2}$ is the least squares solution and satisfies the normal equation $\widetilde{T}_{m}^{T} \widetilde{T}_{m} d^{(j)}=\widetilde{T}_{m}^{T} Q_{m+1}^{T} r^{(j)}$, then $\left(A Q_{m}\right)^{T} \widetilde{r}^{(j)}=0$.

\section{Numerical Experiments}

In order to prove the efficiency of our algorithm, we compare the following methods. (1) MINRES1, it uses MINRES method for solving the multiple right-hand side linear systems one by one and uses the convergent solution of $(j-$ $1)$ th systems as the iterative initial vector of $j$ th systems when the $(j-1)$ th systems converge. (2) MINRES2, it uses
MINRES method for solving the multiple right-hand side linear systems one by one, and the initial vector is zero vector. (3) Mseed is MINRES seed projection method.

All numerical experiments are implemented in MATLAB 2009 and run in Intel Pentium Dual T2390 computer. We set $m=30$; all the tests are stopped as soon as $\left\|r^{(j)}\right\|_{2}<\varepsilon \equiv 10^{-6}$.

Example 1. $A$ is a $1024 \times 1024$ symmetric matrix:

$$
A=\left[\begin{array}{cccccc}
1 & 0.1 & 0.5 & 0.5 & \cdots & 0.5 \\
0.1 & 2 & 0.1 & & & \\
0.5 & 0.1 & 3 & 0.1 & & \\
0.5 & & 0.1 & 4 & \ddots & \\
\vdots & & & \ddots & \ddots & 0.1 \\
0.5 & & & & 0.1 & 1024
\end{array}\right] .
$$

In numerical experiment, the right-hand sides of systems are constructed by two forms as the following, respectively.

Form 1:

$$
\begin{gathered}
B_{1}=\left[b^{(1)}, \ldots, b^{(p)}\right] \\
b^{(j)} \equiv b^{(j)}\left(t_{i}\right)=-\cos \left(5 \cos \left(t_{i}-\frac{2(j-1) \pi}{128}\right)\right),
\end{gathered}
$$

where $j=1, \ldots, p ; t_{i}=1+0.1(i-1), i=1, \ldots, n$.

Form 2:

$$
\begin{array}{r}
B_{2}=\left[b^{(1)}, \ldots, b^{(p)}\right], \quad \text { where } b^{(j)}=A u^{(j)}, u^{(1)}=(1, \ldots, 1)^{T}, \\
u^{(j)}=j \cdot \cos \left((2 j+i) \times 10^{6}\right) \cdot \sin \left((3(4-j)+i) \times 10^{(6)}\right), \\
i=1, \ldots, n, \quad j=2, \ldots, p .
\end{array}
$$

The numerical results are shown in Tables 1 and 2 and the data in bracket is the sum of iterative steps.

In Table 1, the iterative steps of each system are listed and the data in bracket are the sum of iterative steps of all systems. From Table 1, we can know that Mseed can do better than MINRES1 and MINRES2.

Figures 1, 2, and 3 are the convergent curve of three methods, respectively, when the right side is $B_{1}$. And from them, we can see that Mseed can do better than MINRES1 and MINRES2.

Example 2. Next example comes from Matrix Market, and all the matrices are symmetric as shown in Table 3:

$$
B_{1}=\left[b^{(1)}, \ldots, b^{(p)}\right], \quad j=1, \ldots, p,
$$

where $t_{i}=1+0.1(i-1), i=1, \ldots, n, B_{2}=\left[b^{(1)}, \ldots, b^{(p)}\right]$, and $b^{(j)}=(1, \ldots, 1, \underbrace{0, \ldots, 0}_{j-1})^{T}$.

Let $p=5$, and the dimensions of projection space $m=25$ and results of calculation are shown in Table 4.

From Table 4, we can see that Mseed is superior to MINRES method. 
TABLE 1: Iterative steps of convergent solution of each system.

\begin{tabular}{lccc}
\hline Right-hand & Mseed & MINRES1 & MINRES2 \\
\hline$B_{1}$ & $(81) 29,16,8,9,19$ & $(155) 29,20,40,45,21$ & $(286) 29,41,57,73,86$ \\
$B_{2}$ & $(57) 25,15,15,2,0$ & $(125) 25,27,25,21,27$ & $(181) 25,36,34,46,40$ \\
\hline
\end{tabular}

TABLE 2: Convergent time (second) of each method.

\begin{tabular}{|c|c|c|c|}
\hline Right-hand & Mseed & MINRES1 & MINRES2 \\
\hline$B_{1}$ & 17.1720 & 19.2810 & 20.8430 \\
\hline$B_{2}$ & 17.0870 & 18.5620 & 19.7832 \\
\hline \multicolumn{4}{|c|}{ TABle 3} \\
\hline Matrix name & Order & $\begin{array}{l}\text { If diagonal } \\
\text { dominance }\end{array}$ & $\begin{array}{c}\text { Conditions } \\
\text { number }\end{array}$ \\
\hline bcsstm19 & $817 \times 817$ & $\mathrm{Y}$ & $2.3 e+05$ \\
\hline bcsstk27 & $1224 \times 1224$ & $\mathrm{~N}$ & $7.7 e+04$ \\
\hline bcsstk22 & $138 \times 138$ & $\mathrm{~N}$ & $1.7 e+05$ \\
\hline
\end{tabular}

Example 3. $A$ is a $1000 \times 1000$ symmetric matrix:

$$
A=\left[\begin{array}{cccccc}
1 & 0.1 & & & & \\
0.1 & 2 & 0.1 & & & \\
& 0.1 & 3 & 0.1 & & \\
& & 0.1 & 4 & \ddots & \\
& & \ddots & & \ddots & 0.1 \\
& & & & 0.1 & 1024
\end{array}\right]
$$

Form 1:

$$
\begin{gathered}
B_{1}=\left[b_{1}, b_{2}, \ldots, b_{p}\right], \\
\left(b_{j}\right)_{i}=-\cos \left(5 \cos \left(i-\frac{2(j-1) \pi}{128}\right)\right),
\end{gathered}
$$

where $j=1, \ldots, p ; t_{i}=1+0.1(i-1), i=1, \ldots, n$.

Form 2:

$$
\begin{array}{r}
B_{2}=\left[b^{(1)}, \ldots, b^{(p)}\right], \quad \text { where } b^{(j)}=A u^{(j)}, u^{(1)}=(1, \ldots, 1)^{T}, \\
u^{(j)}=j \cdot \cos \left((2 j+i) \times 10^{3}\right) \cdot \sin \left((3(4-j)+i) \times 10^{3}\right), \\
i=1, \ldots, n, \quad j=2, \ldots, p .
\end{array}
$$

The numerical results are shown in Tables 5 and 6 and the data in bracket is the sum of iterative steps.

\section{Conclusion}

In this paper, we propose the MINRES seed projection method for solving symmetric systems with multiple righthand sides, and the residual estimation is analyzed. The numerical examples show that our method is effective.

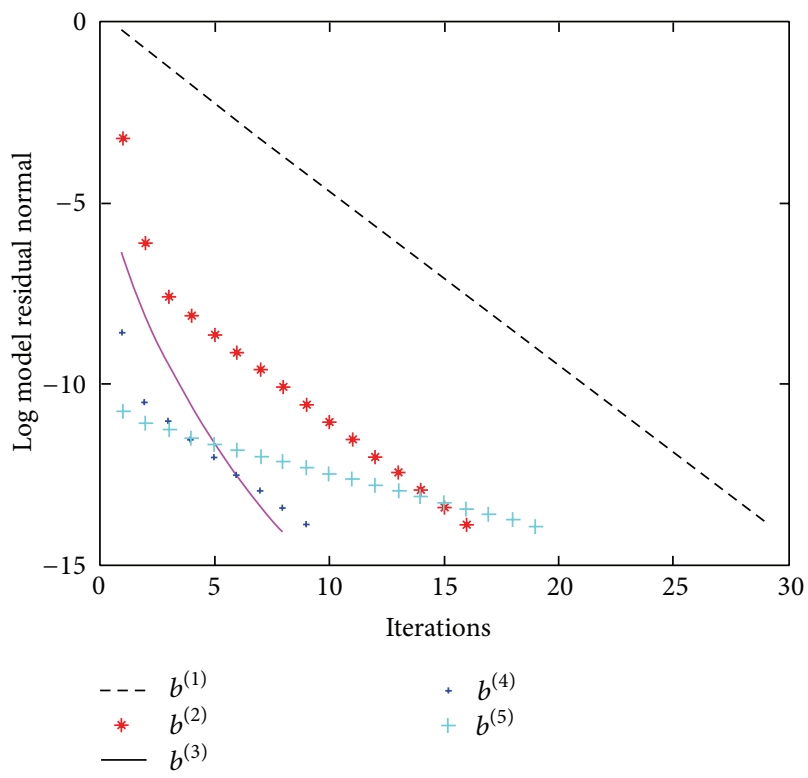

FIGURE 1: MINRES seed projection method.

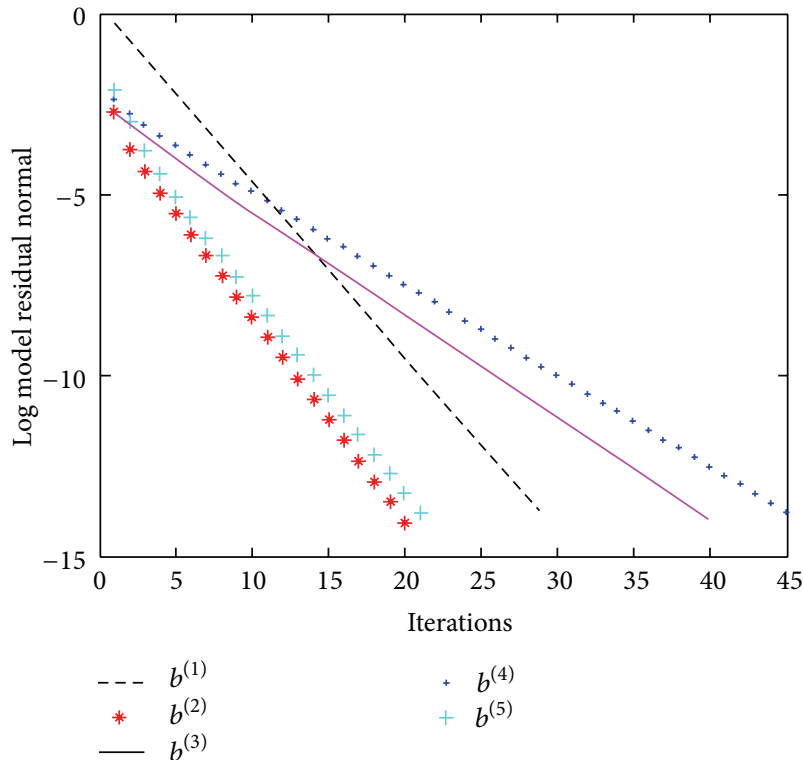

Figure 2: MINRES1.

\section{Conflict of Interests}

The authors declare that there is no conflict of interests regarding the publication of this paper. 
TABle 4: Convergent iterative steps of each system and CPU time (second).

\begin{tabular}{ccccc}
\hline \multirow{2}{*}{$B$} & \multirow{2}{*}{$A$} & \multicolumn{2}{c}{ Minres seed projection method } & \multicolumn{2}{c}{ Minres2 } \\
& Iterative steps & T1 (s) & Iterative steps & T2 (s) \\
\hline \multirow{3}{*}{$B_{1}$} & bcsstm9 & $(718) 171,146,179,129,93$ & 3.13 & $(980) 171,201,241,191,176$ \\
& bcsstk27 & $(781) 211,187,154,124,105$ & 11.82 & $(1033) 211,206,203,203,210$ \\
& bcsstk22 & $(1833) 984,574,142,119,14$ & 4.32 & $(4705) 984,935,850,1014,922$ \\
\hline \multirow{3}{*}{$B_{2}$} & bcsstm9 & $(475) 175,124,62,68,46$ & 2.24 & $(898) 175,201,175,176,171$ \\
& bcsstk27 & $(748) 215,156,143,140,94$ & 11.34 & $(1079) 215,216,216,216,216$ \\
\hline
\end{tabular}

TABLE 5: Iterative steps of convergent solution of each system.

\begin{tabular}{lccr}
\hline Right-hand & Mseed & MINRES1 & MINRES 2 \\
\hline$B_{1}$ & $(34) 11,8,6,4,5$ & $(48) 11,10,9,9,9$ & $(54) 11,11,11,10,11$ \\
$B_{2}$ & $(36) 11,13,11,0,1$ & $(60) 11,11,13,12,13$ & $(60) 11,11,12,13,13$ \\
\hline
\end{tabular}

TABLE 6: Convergent time (second) of each method.

\begin{tabular}{lccc}
\hline Right-hand & Mseed & MINRES1 & MINRES2 \\
\hline$B_{1}$ & 4.785072 & 5.967727 & 6.053866 \\
$B_{2}$ & 4.934010 & 5.739067 & 5.823134 \\
\hline
\end{tabular}

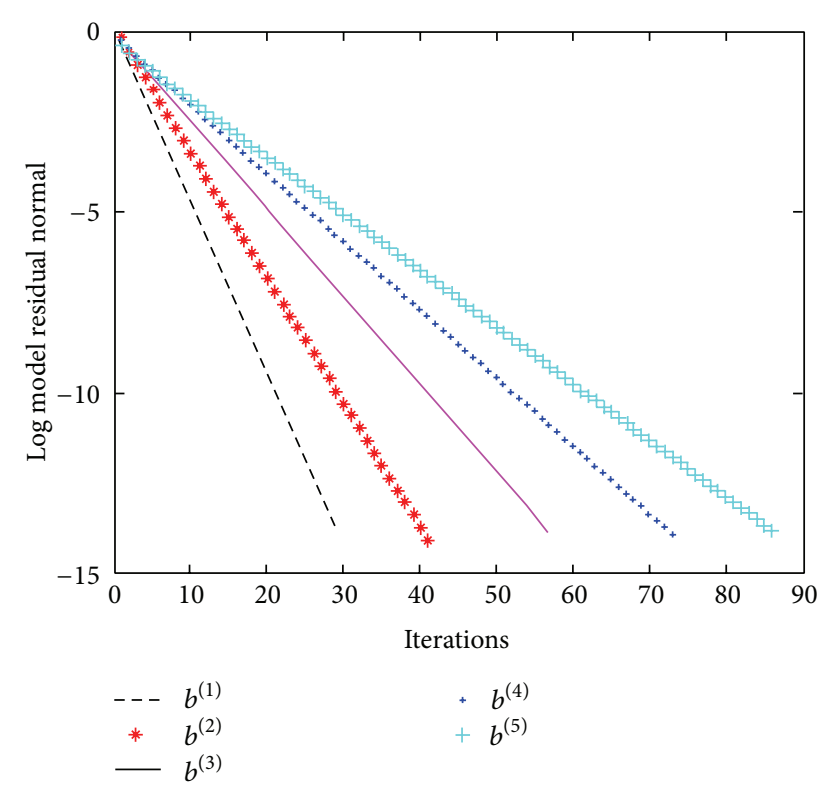

Figure 3: MINRES2.

\section{Acknowledgments}

The authors would like to express their great thanks to the reviewers, who supplied very good opinions about the paper. The work is supported by the Science and Technology Research Project of Education Department of Heilongjiang Province, no. 12521374.

\section{References}

[1] C. F. Smith, A. F. Peterson, and R. Mittra, "Conjugate gradient algorithm for the treatment of multiple incident electromagnetic fields," IEEE Transactions on Antennas and Propagation, vol. 37, no. 11, pp. 1490-1493, 1989.

[2] V. Simoncini and E. Gallopoulos, "An iterative method for nonsymmetric systems with multiple right-hand sides," SIAM Journal on Scientific Computing, vol. 16, no. 4, pp. 917-933, 1995.

[3] D. P. O'Leary, “The block conjugate gradient algorithm and related methods," Linear Algebra and Its Applications, vol. 29, pp. 293-322, 1980.

[4] B. Vital, Etude de quelques méthods résolution de problèmes liné aires de grande taille sur multiprocesseur [Ph.D. thesis], Université de Rennes France, Rennes, France, 1990.

[5] V. Simoncini and E. Gallopoulos, "Convergence properties of block GMRES and matrix polynomials," Linear Algebra and Its Applications, vol. 247, pp. 97-119, 1996.

[6] V. Simoncini and E. Gallopoulos, "Convergence properties of block GMRES and matrix polynomials," Linear Algebra and Its Applications, vol. 247, pp. 97-119, 1996.

[7] A. El Guennouni, K. Jbilou, and H. Sadok, "A block version of BICGSTAB for linear systems with multiple right-hand sides," Electronic Transactions on Numerical Analysis, vol. 16, pp. 129142, 2003.

[8] R. W. Freund and M. Malhotra, "A block QMR algorithm for non-Hermitian linear systems with multiple right-hand sides," Linear Algebra and Its Applications, vol. 254, no. 1-3, pp. 119-157, 1997.

[9] S. Karimi and F. Toutounian, "The block least squares method for solving nonsymmetric linear systems with multiple righthand sides," Applied Mathematics and Computation, vol. 177, no. 2, pp. 852-862, 2006.

[10] A. El Guennouni, K. Jbilou, and H. Sadok, "The block Lanczos method for linear systems with multiple right-hand sides," Applied Numerical Mathematics, vol. 51, no. 2-3, pp. 243-256, 2004.

[11] L. Du, T. Sogabe, B. Yu, Y. Yamamoto, and S.-L. Zhang, "A block $\operatorname{IDR}(s)$ method for nonsymmetric linear systems 
with multiple right-hand sides," Journal of Computational and Applied Mathematics, vol. 235, no. 14, pp. 4095-4106, 2011.

[12] L. W. Zhang, P. Zhu, and K. M. Liew, "Thermal buckling of functionally graded plates using a local Kriging meshless method," Composite Structures, vol. 108, pp. 472-492, 2014.

[13] K. M. Liew, Z. X. Lei, J. L. Yu, and L. W. Zhang, "Postbuckling of carbon nanotube-reinforced functionally graded cylindrical panels under axial compression using a meshless approach," Computer Methods in Applied Mechanics and Engineering, vol. 268, pp. 1-17, 2014.

[14] P. Zhu, L. W. Zhang, and K. M. Liew, “Geometrically nonlinear thermomechanical analysis of moderately thick functionally graded plates using a local Petrov-Galerkin approach with moving Kriging interpolation," Composite Structures, vol. 107, pp. 298-314, 2014.

[15] L. W. Zhang, Z. X. Lei, K. M. Liew, and J. L. Yu, "Large deflection geometrically nonlinear analysis of carbon nanotubereinforced functionally graded cylindrical panels," Computer Methods in Applied Mechanics and Engineering, vol. 273, pp. 118, 2014.

[16] L. W. Zhang, Z. X. Lei, K. M. Liew, and J. L. Yu, "Static and dynamic of carbon nanotube reinforced functionally graded cylindrical panels," Composite Structure, vol. 111, pp. 205-212, 2014.

[17] L. W. Zhang, Y. J. Deng, and K. M. Liew, “An improved elementfree Galerkin method for numerical modeling of the biological population problems," Engineering Analysis with Boundary Elements, vol. 40, pp. 181-188, 2014.

[18] R. J. Cheng, L. W. Zhang, and K. M. Liew, "Modeling of biological population problems using the element-free kp-Ritz method," Applied Mathematics and Computation, vol. 227, pp. 274-290, 2014.

[19] Y. Saad, Iterative Methods for Sparse Linear Systems, SIAM, Philadelphia, Pa, USA, 2nd edition, 2003.

[20] K. Jbilou, A. Messaoudi, and H. Sadok, "Global FOM and GMRES algorithms for matrix equations," Applied Numerical Mathematics, vol. 31, no. 1, pp. 49-63, 1999.

[21] K. Jbilou, H. Sadok, and A. Tinzefte, "Oblique projection methods for linear systems with multiple right-hand sides," Electronic Transactions on Numerical Analysis, vol. 20, pp. 119138, 2005.

[22] M. Heyouni, "The global Hessenberg and CMRH methods for linear systems with multiple right-hand sides," Numerical Algorithms, vol. 26, no. 4, pp. 317-332, 2001.

[23] J. Lai, L. Lu, and S. Xu, "A polynomial preconditioner for the CMRH algorithm," Mathematical Problems in Engineering, vol. 2011, Article ID 545470, 12 pages, 2011.

[24] K. Zhang and C. Gu, "A polynomial preconditioned global $\mathrm{CMRH}$ method for linear systems with multiple right-hand sides," Journal of Applied Mathematics, vol. 2013, Article ID 457089, 7 pages, 2013.

[25] G.-D. Gu, "A seed method for solving nonsymmetric linear systems with multiple right-hand sides," International Journal of Computer Mathematics, vol. 79, no. 3, pp. 307-326, 2002.

[26] G. D. Gu and W. Y. Zhu, "Seed projection methods for solving unsymmetric shifted systems with multiple right-hand sides," Mathematica Numerica Sinica, vol. 26, no. 2, pp. 211-224, 2004. 


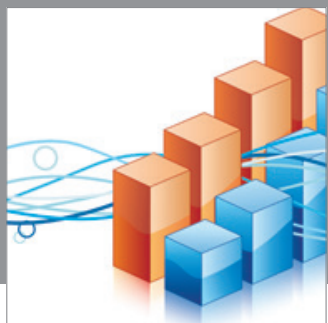

Advances in

Operations Research

mansans

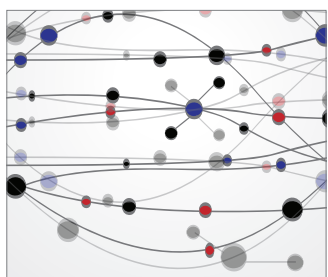

The Scientific World Journal
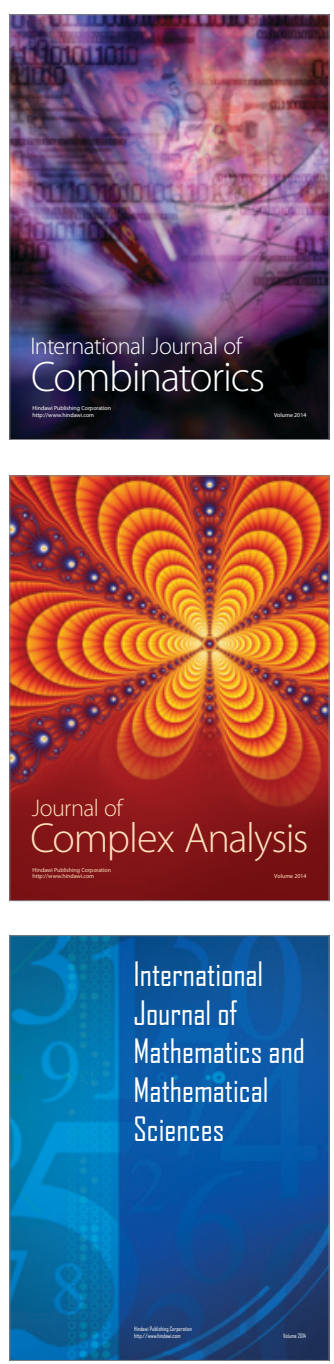
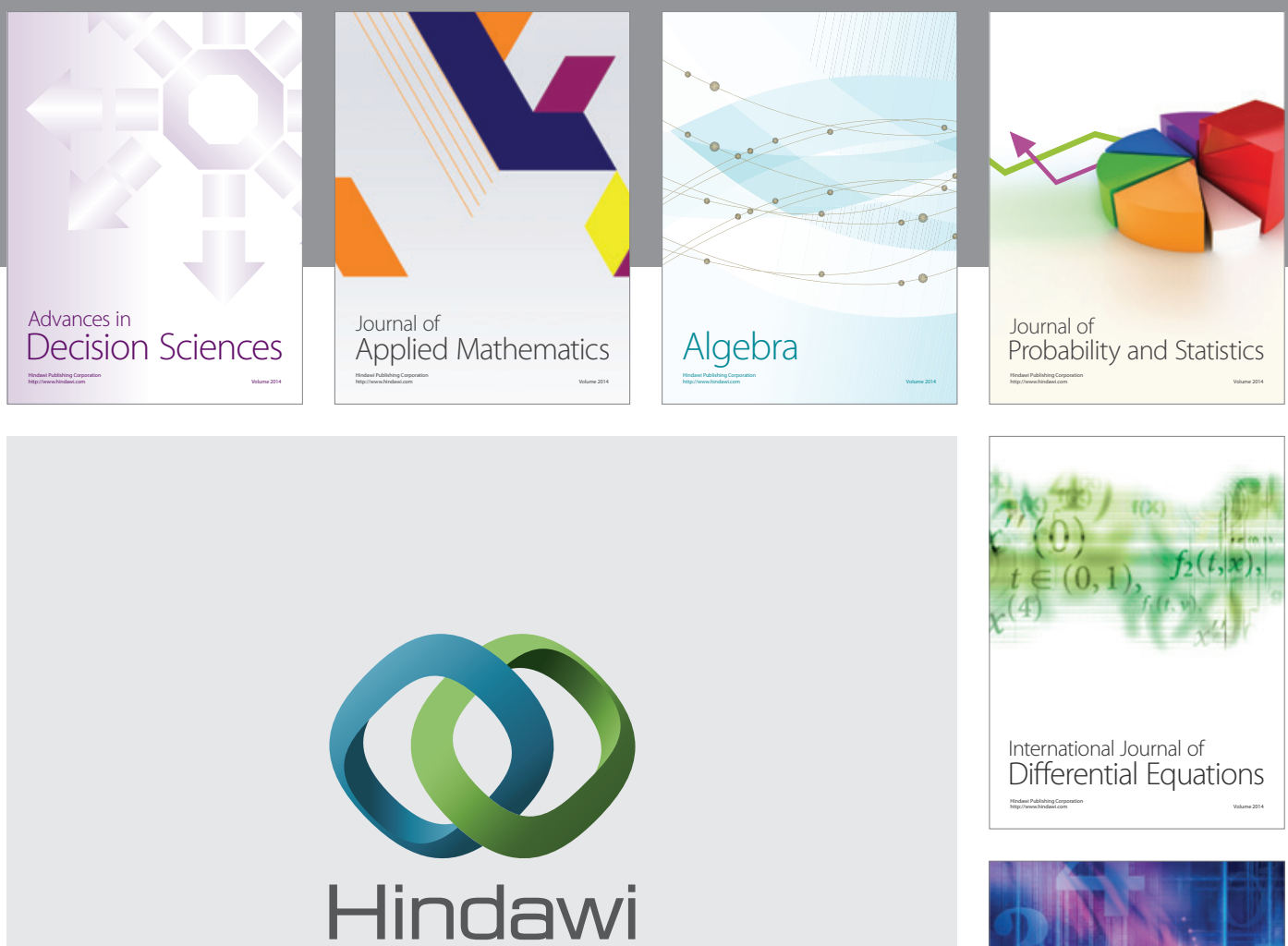

Submit your manuscripts at http://www.hindawi.com
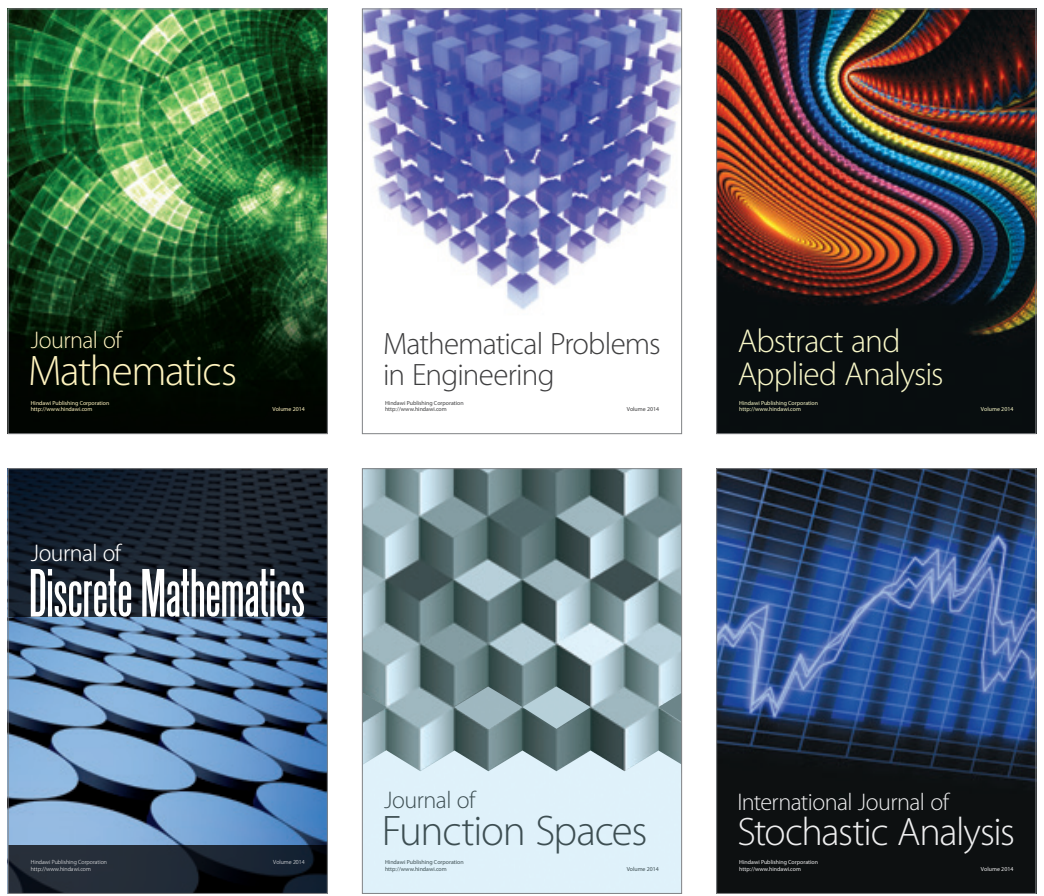

Journal of

Function Spaces

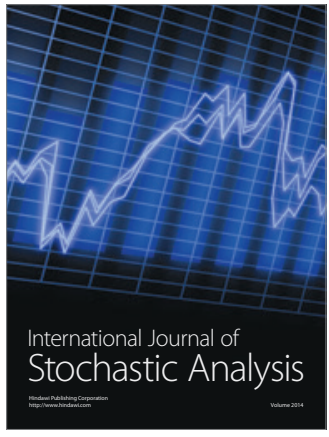

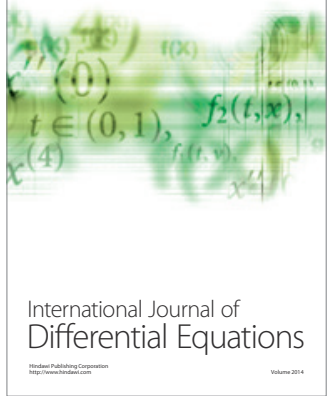
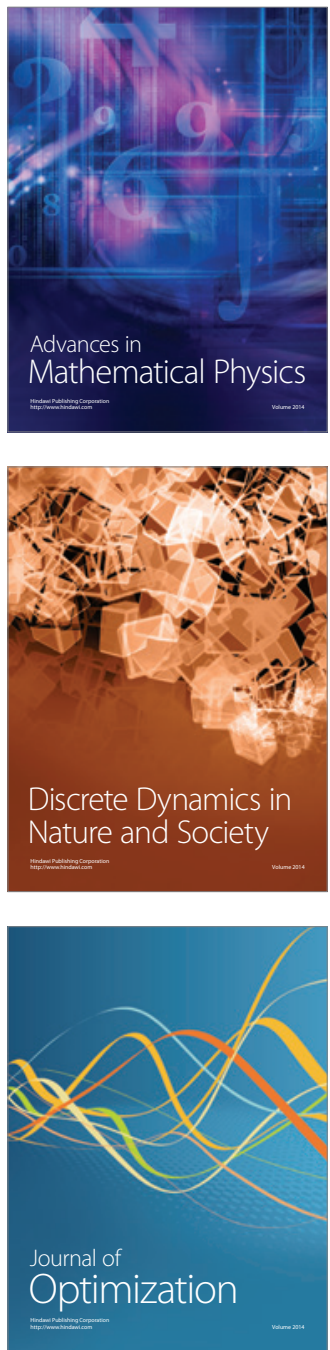